\title{
Modeling the imaging process in optical stellar interferometers
}

\author{
M. Schöller ${ }^{1}$, R. Wilhelm ${ }^{1,2,3}$, and B. Koehler ${ }^{1}$ \\ 1 European Southern Observatory, Karl-Schwarzschild-Str. 2, 85748 Garching b. München, Germany \\ e-mail: mschoell@eso.org, rwilhelm@eso.org, bkoehler@eso.org \\ 2 Deutsches Zentrum für Luft- und Raumfahrt, Institut für Weltraumsensorik, Rutherford-Str. 2, 12489 Berlin, Germany \\ 3 DaimlerChrysler Aerospace, Dornier Satellitensysteme GmbH, SX 2A, 88039 Friedrichshafen, Germany
}

Received December 10, 1999; accepted April 26, 2000

\begin{abstract}
Optical interferometers on the ground, like ESO's Very Large Telescope Interferometer (VLTI) and the Keck Interferometer, and in space, like the InfraRed Space Interferometer (IRSI/Darwin) and the Space Interferometry Mission (SIM), will bring a major breakthrough in optical and near-infrared high angular resolution astronomy at the beginning of the next millennium. These instruments are complex systems with an exceptionally interdisciplinary character involving active/adaptive optics, structural mechanics, control engineering, electronics and various environmental disturbances (e.g. atmospheric turbulence and absorption, wind, seismic noise). For their design and development an approach from two sides is appropriate: laboratory testbeds are used for experimental investigations while numerical modeling tools perform an End-to-End instrument simulation. We have developed a set of numerical modeling tools to simulate the dynamic imaging process of an interferometer. The time-dependent point spread function (PSF) mainly characterizes the imaging performance of the instrument. It is computed by an optomechanical model. Based on the knowledge of the PSF the image of an incoherently radiating extended object is computed using a Fourier optical method. This article describes the modeling approach including an extension to more than two interferometric beams. Some results of simulations on the VLTI as a representative example are shown.
\end{abstract}

Key words: image processing — optical interferometry observational methods - simulation

$\overline{\text { Send offprint requests to: }}$ M. Schöller

\section{Introduction}

Over the last decade optical interferometry has grown out of its pure experimental state. Optical interferometers are running routinely now, producing excellent science (Baldwin et al. 1998; Mourard et al. 1998; Traub 1998; Townes et al. 1998; Johnston 1998; Wallace et al. 1998; Davis et al. 1998). Soon new object classes will become observable with interferometers which provide large apertures as VLTI and Keck (Mariotti et al. 1998; Colavita et al. 1998). Within the next ten years space borne interferometry will offer many advantages including the absence of atmospheric absorption and turbulence, and the possibility to achieve very long baselines (Shao 1998; Penny et al. 1998).

Modern interferometers are highly complex systems combining subsystems of different engineering disciplines, mainly active/adaptive optics, control engineering, electronics and structural mechanics. They typically include a set of nested control loops for various tasks, e.g. image tracking, tip-tilt control, higher-order adaptive optics, fringe tracking, laser metrology or attitude and orbit control system (AOCS). Most of these loops depend intimately on the object observed, and it is very difficult, if not impossible, to calibrate all their effects by observing unresolved stars (Colavita 1999). In combination with experimental work numerical modeling is a powerful technique to study the interactions between the subsystems mentioned above. Furthermore it can be used for preparation of astronomical observations.

The work presented here is a versatile approach to model the imaging process of an interferometer. A computer program package for simulating the dynamic behavior of the VLTI has been developed at ESO. Though targeting mainly at the VLTI the open and flexible structure of the program modules allows their application to a wide range of astronomical interferometers. The total process of imaging an extended astronomical object in the 
aperture synthesis observational mode under the influence of various perturbations can be simulated.

In Sect. 2 we describe the philosophy behind our modeling approach. The description of an interferometer by the incoherent space invariant imaging equation is analyzed, taking pupil mapping under special consideration. Following in Sect. 3 is the description of how the dynamic response of an interferometer is calculated. Building on this result Sect. 4 investigates the computation of the interferometer's point spread function, taking into account multiaxial and coaxial beam combination, and looking into temporal coherence and observation of polarized objects. Imaging of extended objects is explained in Sect.5, followed by some illustrative examples from the model (Sect.6) and the summary (Sect. 7).

\section{Modeling approach}

The modeling approach is based on the incoherent spaceinvariant imaging equation describing the imaging of an incoherently radiating object through an optical system:

$i^{P o l}(\boldsymbol{x})=o^{P o l}(\boldsymbol{x}) * P S F^{P o l}(\boldsymbol{x}) ;$ Pol $\equiv s, p$

where $i^{P o l}(\boldsymbol{x})$ and $o^{P o l}(\boldsymbol{x})$ denote the intensity distributions of the image and object respectively, $P S F^{P o l}(x)$ the point spread function, $\boldsymbol{x}$ a two dimensional vector in image space. The equation holds for the two uncorrelated orthogonal source polarizations of the naturally polarized source specified by the superscript $P o l \equiv s, p$. In the spatial frequency domain the convolution ("*") in Eq. (1) becomes a multiplication:

$I^{P o l}(\boldsymbol{u})=O^{P o l}(\boldsymbol{u}) O T F^{P o l}(\boldsymbol{u}) ;$ Pol $\equiv s, p$

with $I^{P o l}(\boldsymbol{u}), O^{P o l}(\boldsymbol{u}), O T F^{P o l}(\boldsymbol{u})$ being the Fourier transforms of $i^{P o l}(\boldsymbol{x}), o^{P o l}(\boldsymbol{x}), P S F^{P o l}(\boldsymbol{x}) \cdot \boldsymbol{u}$ is a two dimensional vector in Fourier space. The components of $\boldsymbol{u}$ are usually called $u$ and $v$, spanning the $u v$-plane. $O^{P o l}(\boldsymbol{u})$ is the object complex visibility of the object for each source polarization $(\mathrm{Pol} \equiv s, p)$. $O T F^{P o l}(\boldsymbol{u})$ is the optical transfer function $(O T F)$. The $O T F$ is the normalized autocorrelation of the electric field distribution in the exit pupil ("pupil function", see Eq. 8). The modulus of the optical transfer function is called the modulation transfer function (MTF). Equation (2) is the basis for all our modeling algorithms. The calculation of $O^{P o l}(\boldsymbol{u})$ and $O T F^{P o l}(\boldsymbol{u})$ enables us to obtain the fringe pattern as seen by an optical interferometer. Equations (1) and (2) hold for a single wavelength $\lambda$ ("monochromatic light").

Figure 1 illustrates the basic modeling approach. As response to a point source an optomechanical End-to-End model computes the time-dependent electric field distribution ("pupil function") in the exit pupils of the different interferometer arms (at the entrance of the beam combiner). We assume observation of the source in a narrow spectral band (bandwidth $\Delta \lambda \ll$ center wavelength $\lambda$ )

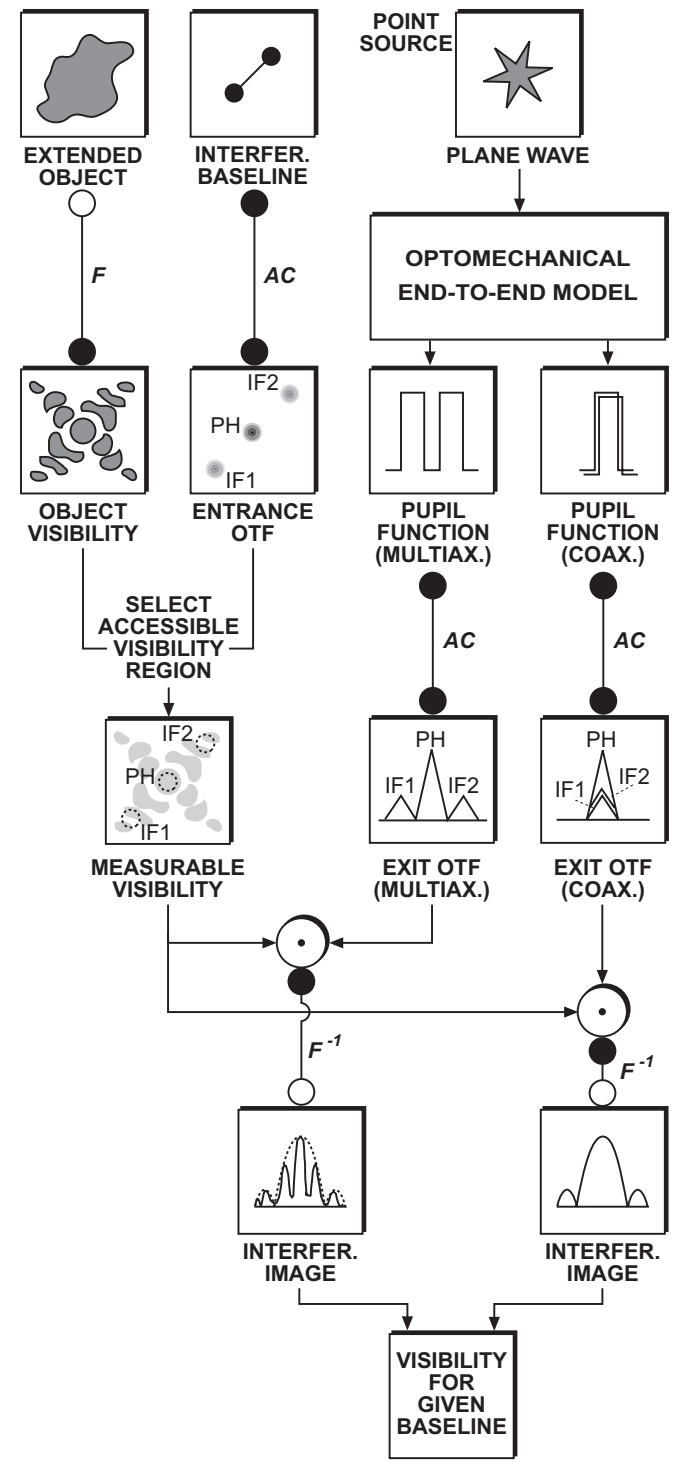

Fig. 1. The modeling approach is based on an optomechanical End-to-End model computing the dynamic instrument response to a point source and a beam combination/imaging model which simulates the imaging of an extended object using a Fourier optical formalism. Note that the peaks in the entrance $O T F$ and in the two exit $O T F$ s have different spacings In case of multiaxial beam combination the three peaks of the exit $O T F$ are not necessarily adjacent to each other. The multiplication of the object complex visibility and the exit $O T F$ is done after shifting the selected visibility regions onto the three $O T F$ peaks appropriately. The interferometric image in the coaxial mode has to be modulated in time to retrieve any object visibility 
("quasimonochromatic approach"). The OTF which in general is wavelength-dependent is computed for the center wavelength of the quasimonochromatic band. The End-to-End model includes models for mechanical structure, control system, environmental disturbances and - as core part - an optical model based on a hybrid ray tracing and diffraction propagation code (Denise \& Koehler 1998; Wilhelm et al. 1998; Wilhelm \& Johann 1999). The output of the optomechanical model serves as input to a separate Fourier optical model which computes the time-dependent optical transfer function at the exit pupil ("exit OTF") for coaxial ("pupil-plane") and multiaxial ("image-plane") beam combination (Schöller et al. 1998). The exit OTF combines the interferometer $O T F$ and atmospheric effects in a single quantity. For a given baseline $\boldsymbol{B}$ (projected perpendicular to the direction of observation) the accessible regions of the object visibility in the spatial frequency domain are determined by the "non-zero" domains of the "entrance $O T F$ " which itself is given by the autocorrelation (denoted by "AC" in Fig. 1) of the electric field distribution in the entrance pupil corresponding to the interferometer baseline ("entrance baseline").

Both, exit and entrance OTF consist of three coneshaped peaks in the spatial frequency plane ("uv-plane"): a central "photometric peak" (denoted by "PH" in Fig. 1) around zero spatial frequency $\boldsymbol{u}=0$ and two "interferometric peaks" (denoted by "IF1" and "IF2" in Fig. 1), symmetric with respect to the origin of the spatial frequency plane. The photometric peak holds only the information of the single apertures, including the total flux. The interferometric peaks hold the interferometric signal, which results from the coherent superposition of the beams related to the two interferometer arms. In the case of a "source visibility" of 1 the interferometric peaks reach their maximum height equal to half the height of the photometric peak, leading to maximum fringe contrast. The interferometric peaks are located at the spatial frequencies corresponding to the entrance and exit baseline, respectively $\left(\boldsymbol{u}= \pm \boldsymbol{B} / \lambda\right.$ for entrance $O T F, \boldsymbol{u}= \pm \boldsymbol{B}^{\prime} / \lambda$ for exit $O T F$ ). The exit baseline $\boldsymbol{B}^{\prime}$ is given by the distance of the two interferometric beams before superposition (in the beam combiner). In case of coaxial beam combination the exit baseline is zero $\left(\boldsymbol{B}^{\prime}=\mathbf{0}\right)$. For a Fizeau-type interferometer which always uses multiaxial beam combination the entrance and exit baseline coincide $\left(\boldsymbol{B}^{\prime}=\boldsymbol{B}\right.$, in all three coordinates $(u, v, w))$, i.e. there is no difference between entrance and exit OTF. On the other hand, in case of a Michelson-type interferometer the exit baseline typically is much shorter than the entrance baseline $\left(\left|\boldsymbol{B}^{\prime}\right| \ll|\boldsymbol{B}|\right)$, hence entrance and exit $O T F$ are different.

The interferometric image for the selected baseline is obtained as follows: The three distinct measurable parts of the object visibility map are multiplied with the corresponding peaks of the exit OTF ("IF1", "PH" and "IF2") (Tallon \& Tallon-Bosc 1992). Inverse Fourier transform of the resulting visibility map yields the interferometric image. For both beam combination modes the envelope of the image is given by the diffraction pattern corresponding to the individual pupils before their combination. While in the multiaxial mode spatial fringes are directly visible in the image a detection in the coaxial mode requires a modulation of the optical path in one interferometer arm to create a temporal fringe pattern. The spacing of the fringes is determined by the exit baseline $\boldsymbol{B}^{\prime}$ or the optical path modulation function for multiaxial or coaxial beam combination, respectively. For both beam combination schemes the fringe contrast is determined by the source visibility map sampled at the spatial frequencies $\pm \boldsymbol{B} / \lambda$. Various algorithms exist for estimating the complex visibility (contrast and phase of the fringe pattern) from a measured spatial or temporal fringe pattern.

The regrouping of the pupils in a Michelson interferometer results in a PSF which is changing over the field-of-view. Tallon \& Tallon-Bosc (1992) describe the object-image relationship for a Michelson interferometer in the monochromatic case. The description of a Michelson interferometer by the incoherent space invariant imaging equation is an approximation which is only correct for a sufficiently small interferometric field-of-view. Outside of this interferometric field-of-view the fringe contrast is deteriorated until there are no fringes anymore. The object intensity distribution has to be convoluted with a field dependent PSF to describe the image intensity distribution. This PSF is not changing in the direction perpendicular to the baseline.

In our simulations we are still using the description by the incoherent space invariant imaging equation, limiting ourselves to a field-of-view with the size of an Airy disk, corresponding to a single subaperture of the interferometer. This is a valid way to describe observations with an interferometer, if the instruments use a spatial filter, thus limiting their field-of-view, anyway. According to Hofmann (1997) (following Tallon-Bosc \& Tallon 1991), the interferometric field-of-view for a given dynamic range is:

$$
F O V=R \frac{\lambda}{B-B^{\prime}} \sqrt{\frac{1}{Q}}
$$

with $R(=\lambda / \Delta \lambda)$ the spectral resolution, $B$ the length of the entrance baseline, $B^{\prime}$ the length of the exit baseline, and $1 / Q$ the dynamic range. One can easily see that the contrast loss can be decreased by using small band filters or dispersion of the signal, enlarging the interferometric field-of-view.

For example, a measurement with the MIDI instrument on VLTI's telescopes UT1 and UT4 (130 m baseline) at $10 \mu \mathrm{m}$ in coaxial beam combination, with a dynamic range of $1 / 100$ and a field-of-view with the size of an Airy disk requires a spectral resolution of $R=150$, smaller than the one which the instrument provides $(R \geq 200)$. 


\section{Computing the dynamic instrument response}

For the simulation of the aperture synthesis imaging process of an interferometer the knowledge of the timedependent optical transfer function $(O T F)$ is of central importance. The $O T F$ is given by the autocorrelation of the pupil function, i.e. the two-dimensional distribution of the complex electric field in the interferometer pupil plane when observing an point source. The task of computing the time-dependent electric field output of the different interferometer arms for a given baseline is performed by an optomechanical End-to-End model (EM). An EM contains models for structural mechanics, control system, sensors, actuators, various perturbations (e.g. atmospheric turbulence, wind), and - as a core part - an optical model which computes the optical signal flow through the instrument. For realistic simulation of the control loop sensors the source intensity in the respective wavelength range has to be taken into account. Modeling the fringe sensor performance requires the knowledge of the object visibility for the given baseline.

\subsection{Modeling interferometer optics}

We have developed a novel optical modeling tool (OM) which is specifically designed to meet the requirements for simulation of ground- and space-based astronomical interferometers (Wilhelm et al. 1998; Wilhelm \& Johann 1999). A fast coordinate-free ray tracing algorithm (Redding \& Breckenridge 1991) forms the basis of the OM. Diffraction propagation is handled either by numerical approximation of the Rayleigh-Sommerfeld integral or by Fourier-optical methods based on the Fast Fourier Transform (FFT). A Jones matrix formalism keeps track of the vectorial electric field. The OM includes a photometry algorithm which calculates the calibrated power flux through the instrument. In contrast to stochastic ray tracing methods of some commercial optical design programs our method makes use of a triangle grid interconnecting the rays. Each triangle is interpreted as an energy-carrying surface element of a locally planar wavefront. Beam propagation is modeled by a sequence of geometrical and physical-optical propagations. The choice of the adequate propagation model (geometri$\mathrm{cal} /$ physical optics) for a single propagation step between two optical surfaces is made with respect to the respective "beam geometry", i.e. the relative sizes of beam diameter, wavefront curvature, optical element aperture and wavelength.

Figures 2 and 3 show scaled models of a VLT Unit Telescope and a VLTI optical delay line, both established with the OM.

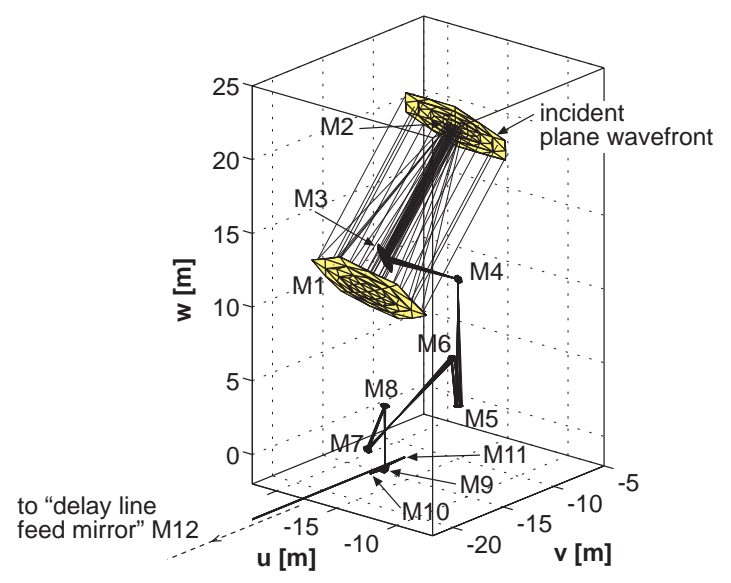

Fig. 2. Scaled model of a VLT unit telescope established with the optical modeling tool. The optical system is subdivided into three groups: (1) telescope optics (M1, M2, M3), (2) Coude train (M4... M8), (3) relay optics (M9... M11). The collimated beam at the telescope output (after reflection at M11) has a diameter of $8 \mathrm{~cm}$. The coordinates $(u, v, w)$ denote the VLTI site coordinates commonly used in ESO publications

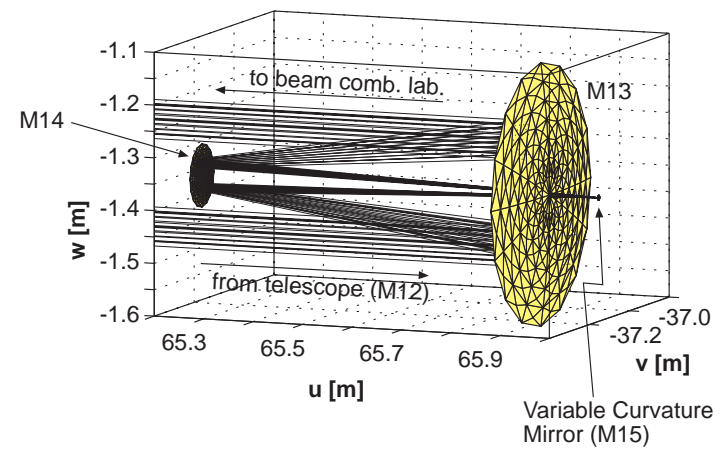

Fig. 3. Scaled model of a VLTI cat's-eye optical delay line established with the optical modeling tool. The optical design is of the "Ritchey-Chretien" type: a telescope with a tertiary mirror at the focus which sends the beam back in a direction parallel to the one it came from. Apart from equalization of the optical path differences the delay line serves the purpose of imaging the exit pupil of a telescope to a fixed location inside the beam combination laboratory. Technically this is accomplished by actively controlling the curvature of the tertiary mirror (M15)

\subsection{Computing the static electric field maps in the pupil plane}

The observation of a point source is simulated by propagating a plane wave through each interferometer arm using a sequence of geometrical and physical-optical propagations. To simulate a naturally polarized starlight beam the computation is done sequentially for two uncorrelated, linearly polarized beams with mutually perpendicular polarization directions (" $s$ " and " $p$ ") defined in the plane 
of the incident wavefront. The vectorial electric field distribution in the pupil plane is computed for each "star polarization" $(s, p)$ :

$\boldsymbol{P}^{P o l}(\boldsymbol{x})=\boldsymbol{E}_{1}^{P o l}(\boldsymbol{x})+\boldsymbol{E}_{2}^{P o l}(\boldsymbol{x}) ;$ Pol $\equiv s, p$

where $\boldsymbol{x}$ denotes a position in the pupil plane and the subscripts "1" and "2" correspond to the two interferometer arms. The function $\boldsymbol{P}^{P o l}$ is the vectorial pupil function which is used to compute the static OTF (see Sect.4).

Figure 4 shows the distribution of the electric field amplitude and phase (one Cartesian component) in the exit pupil plane of a single VLTI arm (one interferometric beam). The field maps result from a hybrid propagation model combining geometrical- and physical-optical propagations. As the propagation of the starlight beam is within the near-field regime the beam diameter at the exit pupil does not substantially deviate from the $8 \mathrm{~cm}$ geometricaloptical footprint (indicated by the white circle in the amplitude map). $92.8 \%$ of the optical power is received within the $8 \mathrm{~cm}$ circular footprint for a wavelength of $2 \mu \mathrm{m}$. On the other hand the amplitude pattern shows significant diffraction effects (typical "near-field ripples"). The phase variation across the $8 \mathrm{~cm}$ diameter corresponds to approximately one wavelength (phase difference $\approx 2 \pi$ ). Outside the $8 \mathrm{~cm}$ circle the slope of the phase map is significantly bigger leading to a rapid oscillation between 0 and $2 \pi$.

\subsection{Computing the time-dependent electric field maps in the pupil plane}

Up to this point we have described the computation of the static electric field distributions. The calculation of the dynamic $O T F_{\mathrm{s}}$ requires the knowledge of the time-dependent electric field distributions $\boldsymbol{E}_{1}^{P o l}(\boldsymbol{x}, t)$ and $\boldsymbol{E}_{2}^{P o l}(\boldsymbol{x}, t)$. Therefore the OM is integrated into an EM. We have implemented the OM described above within the VLTI End-to-End Model, developed at ESO (Denise \& Koehler 1998; Wilhelm \& Koehler 1998). It simulates the dynamic response of the VLTI to a point source. Engineering objectives include the analysis of collective effects of disturbances and the study of interaction of optics and control loops. Eventually, the model is planned to be used as a diagnosis tool during the commissioning of the instrument. Simulated disturbances are wind load on telescopes, atmospheric wavefront piston and tip/tilt, and seismic noise. Atmospheric scintillation and high-spatial frequency corrugations of the wavefront are not taken into account. Mechanical structure modeling is done off-line using finite element software. The response of the interferometer to disturbances is then modeled in the End-to-End model using transfer functions between the disturbances (e.g. wind load) and optical parameters (e.g. optical path length (OPL)). The dynamic control environment model uses a "linear optical model" ("small-motion model"). A linear optical model is represented by a sensitivity matrix

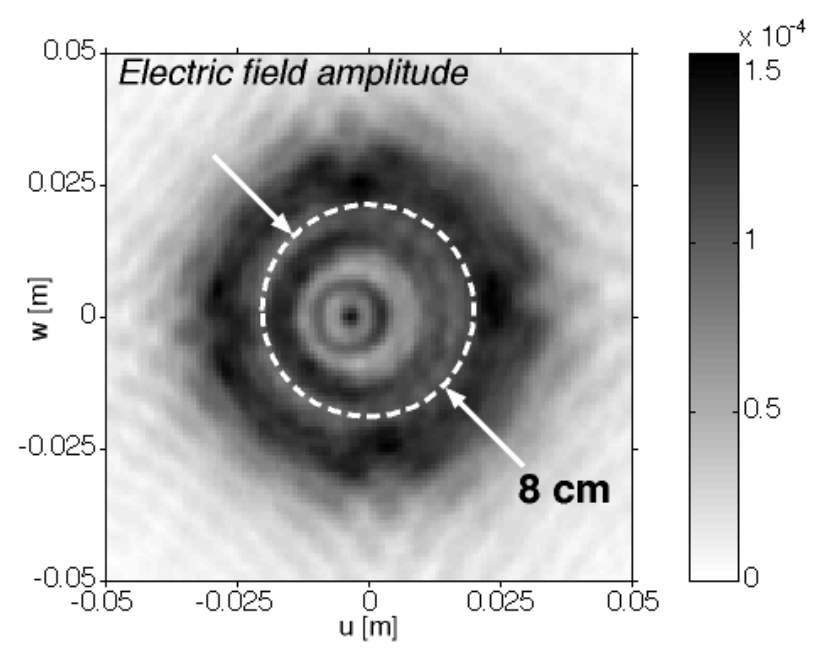

Electric field phase

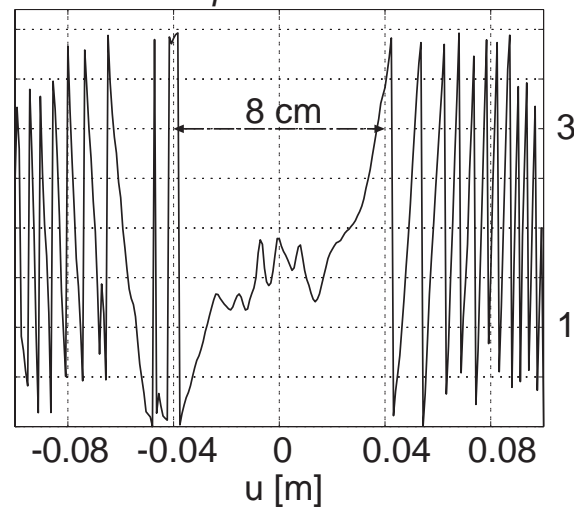

2

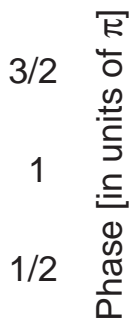

Fig. 4. Field amplitude $[\mathrm{V} / \mathrm{m}]$ (top) and phase [in units of $\pi$ ] (bottom) of one Cartesian component of the complex electric vector field in the exit pupil of a VLTI arm related to a Unit Telescope observing a point source $\left(m_{\mathrm{K}}=10\right)$. The two-dimensional field amplitude distribution is plotted in a $10 \mathrm{~cm} \times 10 \mathrm{~cm}$ square defined in the exit pupil. The phase plot shows a cut through the two-dimensional phase map in the $u$-direction. In both plots the $8 \mathrm{~cm}$ geometrical-optical footprint of the starlight beam is indicated

characterizing the dynamic behavior of a set of optical output parameters in the presence of perturbations acting on the optical system. Examples for optical output parameters are the changes in the position or optical path of a ray or the changes in the Zernike coefficients representing the optical path distribution of a wavefront. The usage of a linear optical model assumes that the perturbations (translations and rotations) of the optical surfaces are small enough to ensure proportionality between the changes in output parameters and the perturbations.

For computation of the dynamic pupil function it is assumed that the time-dependent electric vector field $\boldsymbol{E}_{i}^{P o l}(\boldsymbol{x}, t)$ in the exit pupil of an interferometer arm $i$ is given by the product of a static vector field $\boldsymbol{E}_{i}^{P o l}(\boldsymbol{x})$ and a time-dependent phase factor 


$$
\begin{aligned}
\boldsymbol{E}_{i}^{P o l}(\boldsymbol{x}, t)= & \boldsymbol{E}_{i}^{P o l}(\boldsymbol{x}) \mathrm{e}^{j \Delta \phi_{i}(\boldsymbol{x}, t)} \\
& (i \equiv 1,2 ; \text { Pol } \equiv s, p) .
\end{aligned}
$$

The time-dependent "phase error" $\Delta \phi_{i}(\boldsymbol{x}, t)$ arises from fluctuations of the optical path in the exit pupil with respect to the static situation. As the displacements of the optical elements due to the disturbances and active control are sufficiently small the resulting phase error can be regarded as polarization-independent, i.e. the same factor $\exp \left(j \Delta \phi_{i}(\boldsymbol{x}, t)\right)$ is applied to all Cartesian components of the electric vector field. The equation $\Delta \phi_{i}(\boldsymbol{x}, t)=$ $(2 \pi / \lambda) \Delta O P(\boldsymbol{x}, t)$ links the phase error for a given interferometric arm $i$ to an "optical path error" $\triangle O P$ which itself can be expressed as a Zernike polynomial decomposition using $M$ orthogonal Zernike polynomials $\Psi_{m}$ (typical number $M=15$ ):

$\Delta O P(\boldsymbol{x}, t)=\sum_{m=1}^{M} \mathrm{~d} Z_{m}(t) \Psi_{m}(\boldsymbol{x})$.

The $M$ time-dependent coefficients $\mathrm{d} Z_{m}$ are the deviations of the $M$ Zernike coefficients with respect to the static situation. Within the dynamic simulation the coefficients $\mathrm{d} Z_{m}$ are computed by a linear optical model represented by a sensitivity matrix $\mathbf{A}$ :

$\mathrm{d} \mathbf{Z}(t)=\mathbf{A} * \mathrm{~d} \mathbf{p}(t)$

where $\mathrm{d} \mathbf{Z}(t)$ is a vector of size $M \times 1$ holding the deviations of the $M$ Zernike coefficients, $\mathrm{d} \mathbf{p}(t)$ is a vector of size $6 N \times 1$ holding the perturbations (rotation and translation; 6 degrees of freedom per surface) of the $N$ optical surfaces along a beam train and $\mathbf{A}$ is a $M \times 6 N$ sensitivity matrix. The computation of the sensitivity matrices for the different interferometer arms is done in the preprocessing phase of the EM by sequentially applying small perturbations to the optical surfaces, propagating the starlight beam and watching the changes in the Zernike coefficients in the respective exit pupils.

If the displacement of the exit pupils has to be taken into account appropriate sensitivity matrices can be defined analogously.

\section{The time dependent point spread function of the interferometer}

\subsection{Building the optical transfer function}

This section describes the computation of the point spread function (PSF). As the PSF is the image of a point source the function $o(\boldsymbol{x})$ in Eq. (1) becomes a $\delta$-function corresponding to a constant spectrum $O(\boldsymbol{u})$.

The spacing of the fringes that one obtains with an interferometer depends only on the shape of the exit pupil, or better, its autocorrelation, the $O T F$. In Fig. 5 we show three different one dimensional pupil functions and their
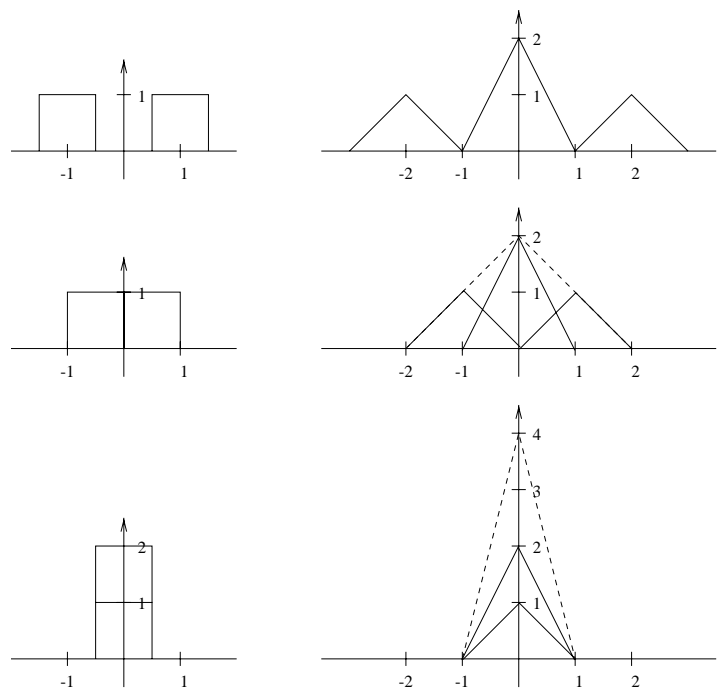

Fig. 5. One dimensional pupil functions (left) and modulation transfer functions (right) for exit pupil separations of 2,1 , and 0 (pupil separation being the distance between the two pupil centers in pupil diameters). The solid lines in the MTFs show the three single parts of the MTF, the dashed lines (where visible) show the total MTF

corresponding MTFs. The first two pupil functions show multiaxial beam combination, the last coaxial beam combination. If the pupils are so close together that the peaks in the $O T F$ are overlapping, the information in the interferometric peaks cannot be fully retrieved. A loss of information in the interferometric peaks means a limit in the interferometric field-of-view. This can be understood if one remembers that an extended object leads to variations on small scales in the object complex visibility, which are not retrievable anymore in the entangled peaks. Using coaxial beam combination, one cannot disentangle the interferometric signal from the photometric signal anymore, since all peaks fall on top of each other (see Sect. 4.3).

In our approach we construct the pupil function and then calculate the $O T F$ from the output of the interferometer simulations described in Sect. 3. Since we must have access to all parts of the OTF separately without mixing them, we are internally computing the $O T F$ always with well separated pupils and rearrange the peaks later to match the real OTF. A simulated two dimensional MTF for image plane beam combination with two telescopes and well separated exit pupils can be seen in Fig. 6 .

The outputs from the optomechanical model are static electric field maps, dynamic Zernike coefficients for the time-dependent OPL maps, and the dynamic lateral pupil positions. For each of the two input polarizations there are three static electric field maps, corresponding to the 


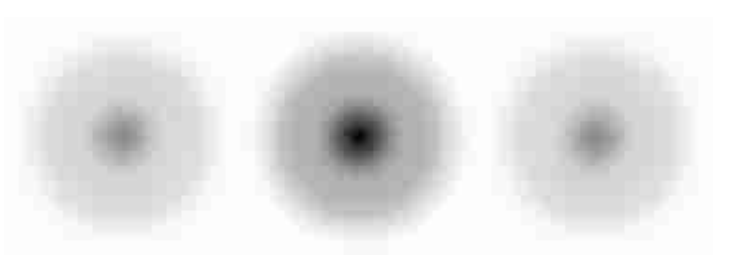

Fig. 6. Modulation transfer function (MTF) for a multiaxial beam combiner. The structure of the MTF is mainly given by the autocorrelation of two uniform disks

three Cartesian components of the electric field ${ }^{\mathrm{a}}$. To reconstruct the exit pupil function from this output for a certain point in time, we take the static electric field maps and multiply them with the dynamic phasors $\exp \left(j \Delta \phi_{i}(\boldsymbol{x}, t)\right)$ (see Eq. 5). To simulate the combination of beams of different sizes (e.g. VLTI beams related to UT and AT) we use a virtual beam expander/compressor adjusting the beams to the same diameter.

From the vectorial pupil function $\boldsymbol{P}^{P o l}$ (Eq. 4) one gets the internally used $O T F^{P o l}$ by computing its autocorrelation (Goodman 1968):

$O T F^{P o l}(\boldsymbol{u})=\frac{\boldsymbol{P}^{P o l}(\lambda \boldsymbol{u}) * \boldsymbol{P}^{P o l *}(\lambda \boldsymbol{u})}{\iint_{-\infty}^{\infty}\left|\boldsymbol{P}^{P o l}(\lambda \boldsymbol{u})\right|^{2} \mathrm{~d} \boldsymbol{u}} ;$ Pol $\equiv s, p$

where $\boldsymbol{u}$ is a two-dimensional, dimensionless vector in the spatial frequency domain $(\boldsymbol{u}=\boldsymbol{x} / \lambda)$. Within the model, the calculation of the $O T F$ is performed using two Fourier transforms:

$O T F^{P o l}=A C\left[\boldsymbol{P}^{P o l}\right]=F\left\{\left|F^{-1}\left\{\boldsymbol{P}^{P o l}\right\}\right|^{2}\right\}$.

The simulation of the imaging process as described in Sect. 2 is done independently for the two star polarizations $(s, p)$ using the two transfer functions $O T F^{s}(\boldsymbol{u})$ and $O T F^{p}(\boldsymbol{u})$.

This way we construct an $O T F_{i}$ for every timestep $t_{i}$ of the dynamic simulation. The length of a single simulation interval $\Delta t=t_{i}-t_{i-1}$ is chosen to ensure stationarity of the atmospheric perturbations. Over an exposure time $T$ we have typically a few $O T F$ s. It is the change of the $O T F$ with time, which is degrading the interferometric signal, and in which we are interested. One gets the $O T F$ for the whole exposure time when averaging the $O T F_{i}$.

Since the dimension of the electric field is $[\mathrm{V} / \mathrm{m}]$, the autocorrelation above has the dimension $\left.\mathrm{V}^{2} / \mathrm{m}^{2}\right]$. One retrieves intensities in $\left[\mathrm{W} / \mathrm{m}^{2}\right]$ when multiplying by $0.5 \sqrt{\mu_{0} / \epsilon_{0}}$ with $\mu_{0}$ and $\epsilon_{0}$ being the dielectric constant and the permeability in vacuum. From here one comes to energies (and finally photons) by multiplying with the area of the beam $A$ and the length in time of the single simulation interval $\Delta t$.

\footnotetext{
a The component in the direction perpendicular to the pupil plane can be neglected as the outcoming beams are close to plane waves.
}

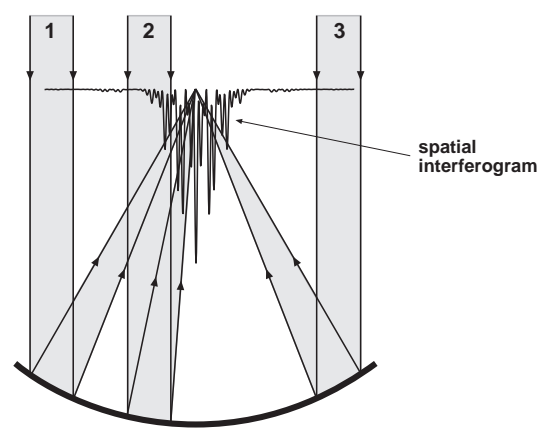

Fig. 7. Multiaxial beam combination with fringe detection in an image plane

The OTF constructed in this way is not dimensionless as described in Eq. (8), but already holds the photometric information. The object complex visibility we are multiplying with this $O T F$ thus has to be dimensionless and normalized to one. Since we are using one object complex visibility for each polarization, the sum of these two visibilities has to be normalized. For an unpolarized object these two visibilities are identical and normalized to 0.5.

Since the OTFs already hold the intensity information, one can directly read the correlated flux for a point source from the $O T F$. It is the sum of the maxima of the interferometric peaks (or twice the height of one peak). The total flux is the maximum of the photometric peak, the uncorrelated flux is then the difference between the total flux and the correlated flux. It should be kept in mind that the two interferometric peaks are symmetric and carry exactly the same information. An extension of the described method to the case of extended objects is given in Sect. 5 .

The combination of more than two beams is performed by using the same method as for two beams. To compute the $O T F$, the $N$ beams have to be arranged in a nonredundant way allowing to retrieve the photometric peak and the $N(N-1) / 2$ interferometric peaks. Afterwards one has to rearrange them according to the exit pupil.

\subsection{Multiaxial ("image plane") beam combination}

For combining the beams in the image plane the exit pupils of two or more interferometer arms are aligned side by side in the pupil plane (see Fig. 7). Imaging this plane by an optical subsystem results in a spatial fringe pattern. The exit pupils have to be aligned so there is no redundancy in the distances between them. Usually the exit pupils are set on a line, with non redundant spacing, leaving the perpendicular axis available for spectroscopy (see Fig. 8). A more compact setup can be achieved by using two dimensions, taking for example a trapezium structure with four exit pupils (Fig. 9). 


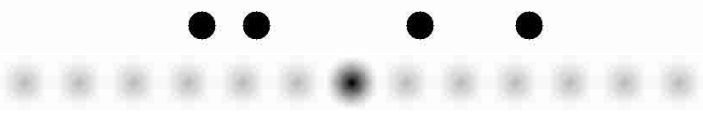

Fig. 8. Non-redundant pupil function and the corresponding modulation transfer function for four beams and a linear setup
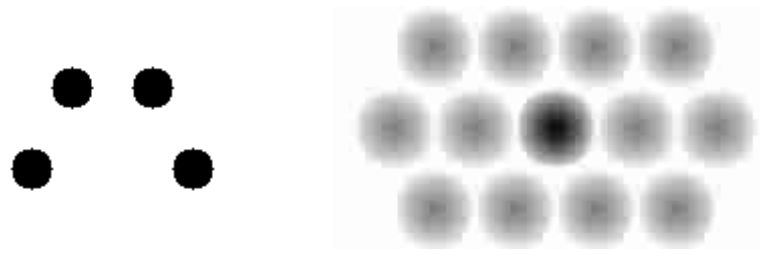

Fig. 9. Non-redundant pupil function and the corresponding modulation transfer function for four beams and a compact setup

When observing in multiaxial mode one frame per atmospheric coherence time is taken. A usual method for estimation of the visibility amplitude $V$ for an interferometer combining two beams, which have been spatially filtered to the extent of a single aperture Airy disk, is given in Roddier \& Léna (1984): By taking the Fourier transform and squaring it one computes the power spectrum (squared MTF) of the spatial fringe pattern. The power spectrum has non-overlapping low and high-spatial frequency terms (the single photometric peak and the two interferometric peaks). The ratio of the high to low frequency energies gives a good estimation of the term $\frac{1}{2} V^{2}$.

For observations with a field-of-view exceeding the size of an Airy disk, one has to evaluate the values at every single spatial frequency within the interferometric peaks of the power spectrum.

This mode will be used by the AMBER beam combiner for the VLTI (Petrov et al. 1998). In Fig. 10 a simulated image plane combined interferogram can be seen.

\subsection{Coaxial ("pupil plane") beam combination}

Most interferometers are combining the beams coming from the arms of the interferometer in the pupil plane. This means that the exit pupils are aligned pairwise on top of each other. Afterwards usually an optical subsystem focuses the pupil on a detector (see Fig. 11).

To measure the fringe, the observer has to step through the fringe in time, namely modulating the optical path in one of the interferometer arms. One way to achieve this is to take four frames per atmospheric coherence time, each of which has $(0,1,2,3) \times \lambda / 4$ optical path difference (OPD) added to one beam. The visibility is estimated by determining the energy in each of the four frames $(A, B, C, D)$ and computing the modulus of the object complex visibility according to: $\frac{1}{2} \sqrt{(A-C)^{2}+(D-B)^{2}}$.

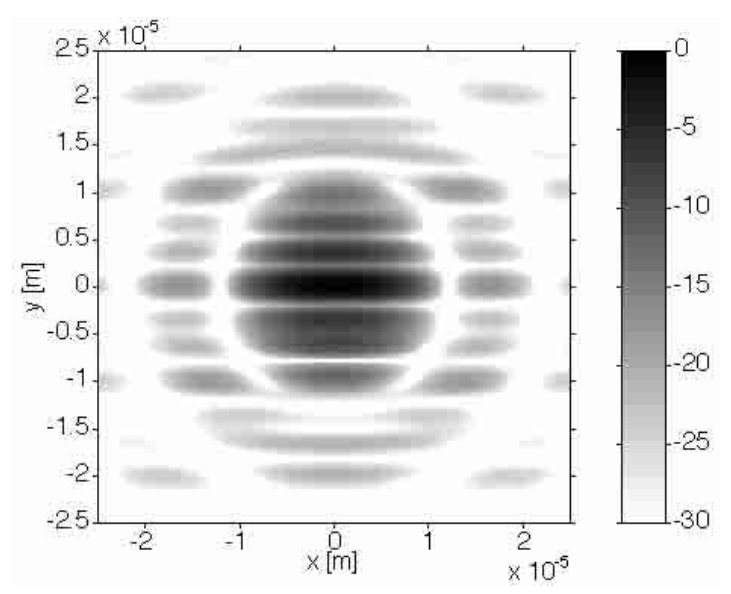

Fig. 10. Image plane combined interferogram as observed in the focal plane of a Fizeau interferometer with three circular $1.8 \mathrm{~m}$ subapertures (wavelength $550 \mathrm{~nm}$, effective focal length $34.65 \mathrm{~m}$, logarithmic dB-scale (relative to maximum)). The baselines have a length of $1.8 \mathrm{~m}, 3.6 \mathrm{~m}$, and $5.4 \mathrm{~m}$

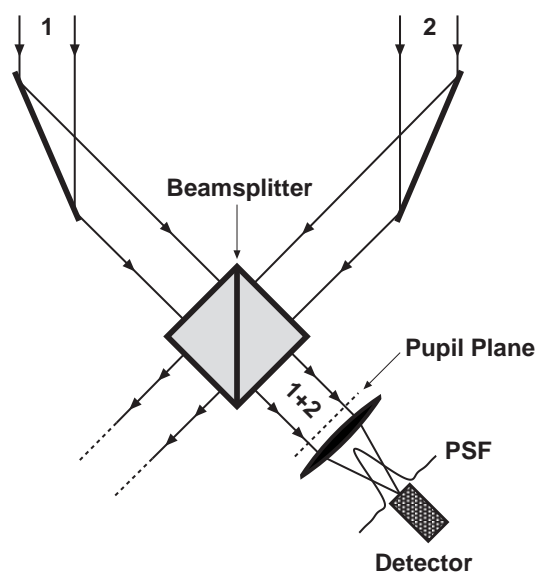

Fig. 11. Coaxial beam combination with fringe detection in an image plane

The phase of the object complex visibility can also be computed from the four energy bins $A, B, C, D$ (Shao \& Staelin 1977).

For the beam combination in the model this means that one has to add $0, \pi / 2, \pi, 3 \pi / 2$ to the phase in one of the interferometric $O T F$ peaks, and subtract the same value in the other interferometric peak. Figures 12 and 13 illustrate what consequence this additional phase has on the $O T F$.

This beam combination mode is foreseen with the MIDI instrument for VLTI (Leinert \& Graser 1998). Four Airy disks of varying intensities, which form a typical interferogram can be seen in Fig. 14.

When combining more than two beams in coaxial beam combination it is necessary to sample the fringe with more than four steps in time. This leads to different steps in 

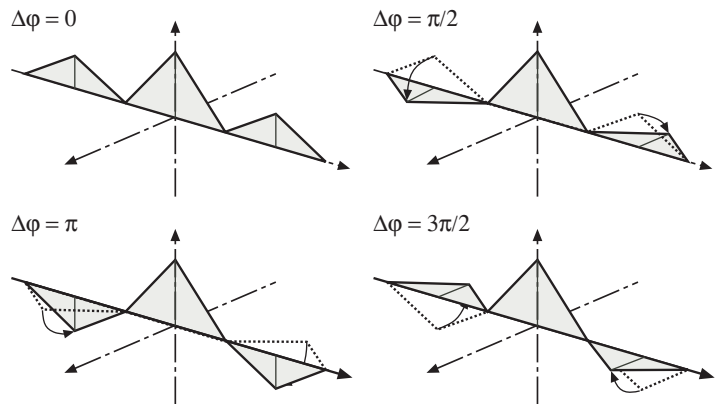

Fig. 12. Optical transfer function for two rectangular apertures (multiaxial beam combination, exit baseline has the length of twice the pupil diameter), where a phase of $0, \pi / 2, \pi$, and $3 \pi / 2$ is added to one of the beams. The interferometric peaks of the $O T F$ are rotating around the complex axis. (Shown just for illustration since there is no path modulation necessary in multiaxial beam combination)
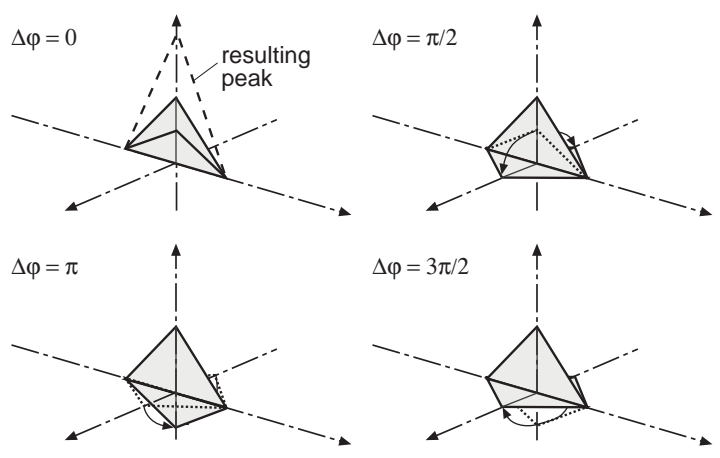

Fig. 13. Same as Fig. 12, but for a coaxial beam combination. In the upper left the two interferometric peaks are enhancing the height of the photometric peak (no phase shift), in the two right drawings the interferometric peaks cancel, leaving only the photometric peak (phase shifts $\pi / 2$ and $3 \pi / 2$ ), while in the lower left the two interferometric peaks cancel the photometric peak, leaving no signal (phase shift $\pi$ )

phase for the interferometric peaks, which are now rotating around the imaginary axis with different speed. One of the beams is not delayed at all as another beam has to be delayed by steps of $\lambda / 4$, just like in the case of two beam combination. The third beam has to be delayed with steps of $\lambda / 12$. A total of 12 exposures has to be taken. For the six interferometric peaks of the $O T F$ follows that two have to rotate by steps of $\pi / 2$, two with $\pi / 3$, and the last two with $\pi / 6$. The first make three rotations during one fringe acquisition, the second two rotations, and the latter one rotation.

\subsection{Taking temporal coherence into account}

Within the modeling software we assume the validity of the quasi monochromatic approximation. A finite band-

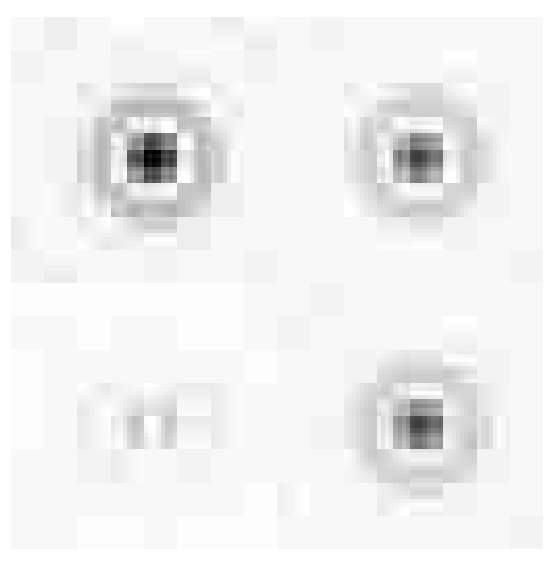

Fig. 14. Pupil plane combined interferograms at $10 \mu \mathrm{m}$ for delays of $0, \lambda / 4, \lambda / 2$, and $3 \lambda / 4$ (square root display). The lower left interferogram should be 0 over the whole image for a perfect interferometer and an unresolved object. This is not the case because of non-zero optical path difference (OPD) between the two arms of the interferometer and optical aberrations. Since the interferometric information is the total intensity on the detector, in real applications only one pixel is needed to record the data

width of the source which is much smaller than the center wavelength/frequency can however be taken into consideration. This leads to a degradation of the visibility. In the picture of correlated versus uncorrelated flux this means that the correlated flux becomes less. Thus one has to lower the interferometric peaks in the $O T F$ by a generally complex factor $\gamma(0 \leq|\gamma| \leq 1) \cdot \gamma$ is the degree of temporal coherence. It is a function of the OPD between the two interfering wavefronts and is computed as the Fourier transform of the power spectral density of the source (Goodman 1985). As a first approach we average the OPD between the beams across their areas and multiply the interferometric peaks in the $O T F$ with this value. If the average OPD substantially exceeds the coherence length of the starlight the degradation factor $|\gamma|$ becomes zero and the fringes disappear. This computing approach does not consider a variation of the OPD across the beam areas.

\subsection{Simulating polarized objects}

A polarized object can be characterized in every pixel of the intensity distribution by three quantities: the flux, the degree of polarization, and the direction of polarization. In the model the polarized and the unpolarized flux are separated. The polarized flux is projected on the two polarization axes used within the optomechanical model. The unpolarized flux of the object is divided by two and added to the two polarization maps. From these two maps we calculate two object complex visibilities. Both are multiplied 


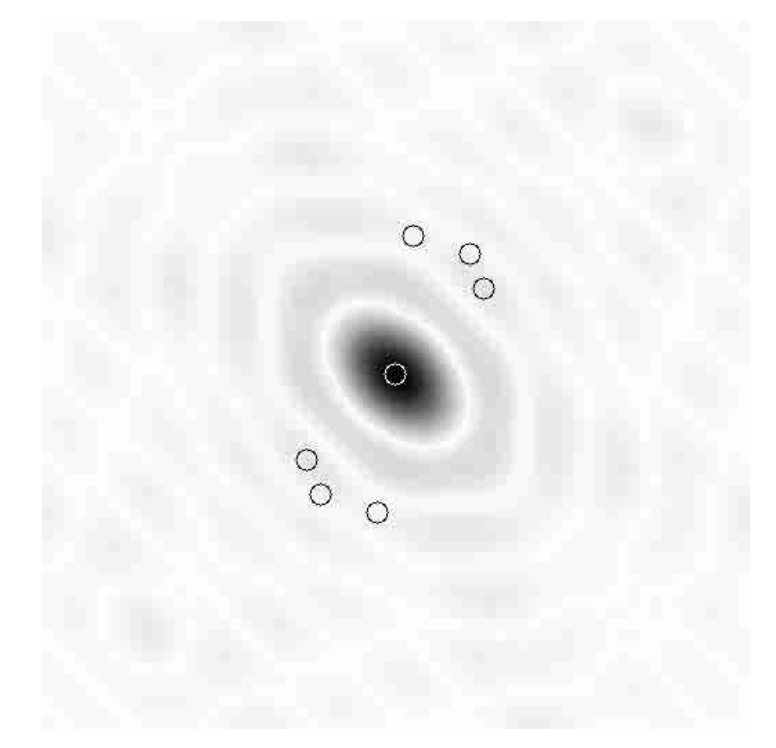

Fig. 15. Modulus of the object complex visibility of a uniform ellipse. The encircled areas are the parts of the object complex visibility picked up by VLTI's UT1 and UT3 for an object at declination $-30^{\circ}$ and hour angles $-3,0$, and 3

with their corresponding $O T F \mathrm{~s}$. Now the two different visibilities have to be added to retrieve the measured signal.

\section{Imaging extended objects}

As soon as the individual apertures are not placed on a single steering mount, the entrance pupil of earth bound interferometers is different for objects with different declinations and varies with hour angle. Thus the part of the object complex visibility which is measured with the interferometer varies with time (see Fig. 15).

From the object complex visibility we have to cut out the parts which are picked up by the entrance pupil of the interferometer. These are just the parts which lie in the $u v$-coverage belonging to the current array configuration for the observed object. The object complex visibility patches are now multiplied on their respective parts in the $O T F$, as illustrated in Fig. 1.

For an earth-bound interferometer the baseline is changing with time with respect to the object. Thus the interferometer is sensitive to different parts of the object complex visibility. The projected baseline of an earth-bound interferometer can be calculated by

$(u, v, w)=\operatorname{Rot}(x, \delta) \operatorname{Rot}(y, H) \operatorname{Rot}(x, l)(x, y, z)$

with $\operatorname{Rot}(a, \alpha)$ being a rotation around axis $a$ with angle $\alpha, \delta$ the declination of the object, $H$ the hour angle of the observation, and $l$ the latitude of the observatory. $x$ is pointing to the east, $y$ to the north, and $z$ to zenith. $u$ and $v$ are coordinates in the $(u, v)$-plane and $w$ is giving the sidereal optical path delay.
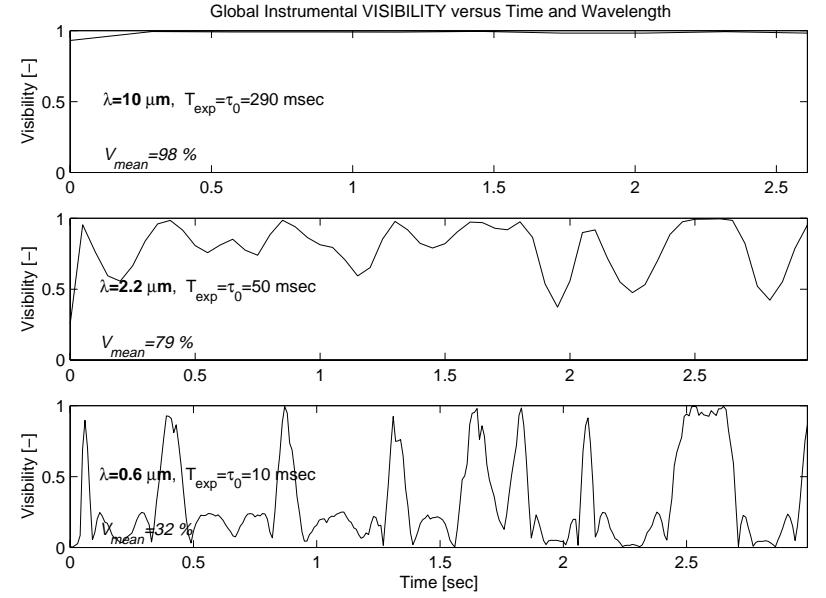

Fig. 16. Time-dependent visibility amplitude computed for an idealized pupil plane instrument combining two beams of the VLTI. The visibility has been computed for three different wavelengths $(10 \mu \mathrm{m}, 2.2 \mu \mathrm{m}$, and $0.6 \mu \mathrm{m})$ with the exposure times $T_{\exp }$ matching the atmospheric coherence time $\tau_{0}$ for each wavelength $\left(\tau_{0}=290 \mathrm{~ms}, 50 \mathrm{~ms}, 10 \mathrm{~ms}\right.$, respectively)

A value of the object complex visibility is estimated for every timestep of the aperture synthesis imaging process, i.e. for every orientation of the interferometer baseline with respect to the observed object. Using the Van Cittert Zernike theorem the object intensity distribution (in the sky) can be partially reconstructed from the measured visibilities.

\section{Illustrative examples from the model}

In this section we show some results of the simulations of the VLTI. These are based on ESO's optomechanical End-to-End model.

Figure 16 shows the global instrumental visibility as measured by an idealized pupil plane instrument. The visibility measured by a true instrument will be severely effected by thermal background at $10 \mu \mathrm{m}$ and high spatial frequency wavefront corrugations due to the turbulent atmosphere at the shorter wavelengths which are both not included here. The peaks with a period of 0.5 seconds in the $0.6 \mu \mathrm{m}$ visibilities arise from the residual tracking errors of the telescopes under wind load, which lets the two Airy disks overlap only from time to time. These residual errors can be corrected by increasing the gain of the fast tracking loop which has been set to low values here on purpose. The first $100 \mathrm{~ms}$ of each time series are dominated by the model initialization phase and are not usable.

Residual tilt is dominating the degradation of the visibility at short wavelengths. Figure 17 shows the effect of residual tilt present in the exit pupil plane on the resulting interferometric image for three different wavelengths. While for $10 \mu \mathrm{m}$ the Airy disks overlap perfectly, at $2 \mu \mathrm{m}$ 


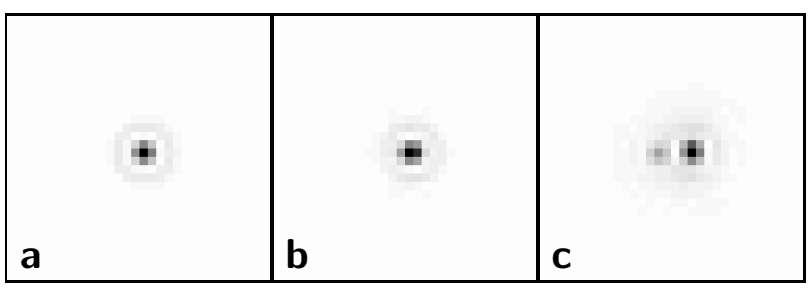

Fig. 17. Coaxially combined interferograms at a) $10 \mu \mathrm{m}$, b) $2 \mu \mathrm{m}$, and c) $500 \mathrm{~nm}$ (see text)

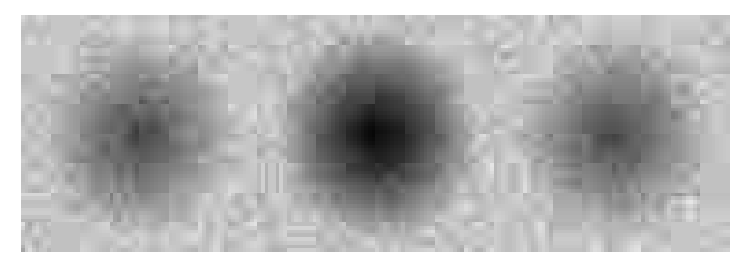

Fig. 18. Fourier spectrum of an image plane combined interferogram at $2 \mu \mathrm{m}$ with 10000 photons and $20 \mathrm{e}^{-}$readout noise (square root display)

one can see a slight elongation, and for $500 \mathrm{~nm}$ the Airy disks are separated.

Another aspect that we studied is the influence of the detector when using the interferometer at low light levels. We added noise to a multiaxially combined interferogram, corresponding to 10000 photons and $20 \mathrm{e}^{-}$readout noise. In Fig. 18 the modulus of the corresponding Fourier spectrum can be seen. The interferometric peaks which carry the signal we want to measure are blurred, thus degrading the visibility significantly.

\section{Summary and outlook}

The purpose of this article is to present a powerful and versatile approach to dynamic modeling of the imaging process of an optical stellar interferometer operating in aperture synthesis mode. Therefore a set of numerical models has been developed and linked together to form a comprehensive analysis tool covering the various engineering disciplines of astronomical interferometry. Although most of the model components were specifically developed for the VLTI their modular and open structure allows their application to other related instruments. The modeling tool can be divided into two main components: (1) An optomechanical End-to-End model computes the time-dependent instrument response to an point source, i.e. the complex electric field distribution at the entrance of the interferometric beam combiner. This serves as input to (2) a Fourier optical model which simulates the imaging of extended objects. The final output of the complete model is the dynamic complex fringe visibility for a given baseline. The article describes the modeling approach il- lustrated by examples and some didactic simulation results obtained for the VLTI.

Future work intends a refinement of the optical modeling algorithms including support for deformable/segmented mirrors (adaptive optics) and further enhancement of the models for diffraction propagations. An atmospheric turbulence model will be included to supplement the existing disturbance models for atmospheric wavefront piston and tip/tilt, wind load and seismic noise. The various optical detectors and sensors have to be modeled in a realistic way taking sensitivity and noise aspects into account.

Eventually the program package can be useful during all preparatory and operational phases of a stellar interferometry project. In the planning phase it can be used for detailed demonstration of instrument feasibility. During the design and analysis phase the program enables a gradual refinement of the subsystem layouts (control, optics, structure) by studying their mutual interaction. Finally, in the operational phase it can be used for preparation of observations. A preceding simulation run can determine optimum values of instrument parameters (e.g. baseline configuration, integration time) for a given scientific objective.

Acknowledgements. We would like to thank Andreas Glindemann, Thilo Hannemann, and Pierre Kervella for fruitful discussions, and the anonymous referee for valuable comments. This work has been supported in part by the German Aerospace Center DLR (Dr. E. Bachem) under contract OISI FKZ 50 TT 9439.

\section{References}

Baldwin J.E., Boysen R.C., Haniff C.A., et al., 1998, in: Reasenberg R.D. (ed.) Proc. SPIE 3350, Astronomical Interferometry, p. 736

Colavita M.M., Boden A.F., Crawford S.L., et al., 1998, in: Reasenberg R.D. (ed.) Proc. SPIE 3350, Astronomical Interferometry, p. 776

Colavita M.M., 1999, PASP 111, 111

Davis J., Tango W.J., Booth A.J., O’Byrne J.W., 1998, in: Reasenberg R.D. (ed.) Proc. SPIE 3350, Astronomical Interferometry, p. 726

Denise C., Koehler B., 1998, VLTI End-to-End model user guide, Tech. Rep. VLT-MAN-ESO-15000-1569, European Southern Observatory

Goodman J.W., 1968, Introduction to Fourier Optics. McGraw-Hill, New York

Goodman J.W., 1985, Statistical Optics. John Wiley \& Sons, Inc., New York

Hofmann K.-H., 1997, ISAC Meeting \#5, European Southern Observatory

Johnston K.J., 1998, in: Reasenberg R.D. (ed.) Proc. SPIE 3350, Astronomical Interferometry, p. 941

Leinert C., Graser U., 1998, in: Reasenberg R.D. (ed.) Proc. SPIE 3350, Astronomical Interferometry, p. 389 
Mariotti J.-M., Denise C., Dérie F., et al., 1998, in: Reasenberg R.D. (ed.) Proc. SPIE 3350, Astronomical Interferometry, p. 800

Mourard D., Thureau N., Antonelli P., et al., 1998, in: Reasenberg R.D. (ed.) Proc. SPIE 3350, Astronomical Interferometry, p. 517

Penny A.J., Leger A., Mariotti J.-M., et al., 1998, in: Reasenberg R.D. (ed.) Proc. SPIE 3350, Astronomical Interferometry, p. 666

Petrov R., Malbet F., Richichi A., Hofmann K.-H., 1998, The ESO Messenger 92, 11

Redding D.C., Breckenridge W.G., 1991, J. Guid. Contr. Dyn. 14,1021

Roddier F., Léna P., 1984, J. Opt. 15, 171

Schöller M., Denise C., Koehler B., 1998, in: Reasenberg R.D. (ed.) Proc. SPIE 3350, Astronomical Interferometry, p. 818

Shao M., Staelin D.H., 1977, J. Opt. Soc. Am. 67, 81

Shao M., 1998, in: Reasenberg R.D. (ed.) Proc. SPIE 3350, Astronomical Interferometry, p. 536

Tallon M., Tallon-Bosc I., 1992, A\&A 253, 641

Tallon-Bosc I., Tallon M., 1991, in: Proc. ESO Conf., High Resolution Imaging by Interferometry II, p. 805

Townes C.H., Bester M., Danchi W.C., et al., 1998, in:
Reasenberg R.D. (ed.) Proc. SPIE 3350, Astronomical Interferometry, p. 908

Traub W.A., 1998, in: Reasenberg R.D. (ed.) Proc. SPIE 3350, Astronomical Interferometry, p. 848

Wallace J.K., Boden A.F., Colavita M.M., et al., 1998, in: Reasenberg R.D. (ed.) Proc. SPIE 3350, Astronomical Interferometry, p. 864

Wilhelm R., Johann U., Schalinski C., Jahn H., 1998, in: Reasenberg R.D. (ed.) Proc. SPIE 3350, Astronomical Interferometry, p. 282

Wilhelm R., Denise C., Koehler B., Schöller M., 1998, in: Abstracts of Contributed Talks and Posters presented at the Annual Scientific Meeting of the Astronomische Gesellschaft at Heidelberg, September 14-19, 1998, Astronomische Gesellschaft Meeting Abstracts, talk \#L4

Wilhelm R., Koehler B., 1998, BeamWarrior optical kernel and its implementation within the VLTI End-to-End Model, User Manual VLT-MAN-ESO-15000-1673, European Southern Observatory

Wilhelm R., Johann U., 1999, in: Merkle F. (ed.) Proc. SPIE EUROPTO Series 3737, Design and Engineering of Optical Systems, p. 45 